\title{
ROLE OF MULTIDETECTOR COMPUTED TOMOGRAPHY SCAN IN THE ASSESSMENT OF MEDIASTINAL MASSES
}

\author{
HIMAL RAI ${ }^{1}$, MANNA DEBNATH ${ }^{2 *}$, ANAND P VERMA ${ }^{3}$ \\ ${ }^{1}$ Department of Medical Imaging Technology, Assam Downtown University, Assam, India. ${ }^{2}$ Department of Medical Imaging \\ Technology, Charotar Institute of Paramedical Sciences, Charotar University of Science and Technology, Changa, Gujarat, \\ India. ${ }^{3}$ Department of Radiodiagnosis and Imaging, National Institute of Medical Sciences and Research, Jaipur, Rajasthan, \\ India. Email: mannadebnath93@gmail.com
}

Received: 07 May 2020, Revised and Accepted: 18 June 2020

\section{ABSTRACT}

Objectives: The objectives of the study were to determine the sensitivity and specificity of multidetector computed tomography (MDCT) in the evaluation of masses present in the mediastinum and to determine the localization and the common age group exposed to mediastinal masses.

Methods: A retrospective cross-sectional study included 33 males and 21 females' samples in the age range between 20 to 70 years. The patients who are specifically suspected of mediastinal masses were included in this research study and accumulated the data. The research was conducted between August 2017 to March 2018. The analysis of the data was performed using Microsoft Excel 2010.

Results: Patients were referred for MDCT scan of the chest to the department of radiodiagnosis with a clinical suspicion of a mediastinal mass or who had an abnormal chest radiograph suggestive of mediastinal mass during the study period. The final result of the study clearly indicates that the majority of the patients that fall within the age group of 40-49 years and 50-59 years have the highest probability of occurring mediastinal masses.

Conclusion: The present study concluded that anterior mediastinum is the most usual compartment involving the formation of mediastinal mass, followed by the posterior and middle compartment. MDCT is the modality of choice, which can evaluate mediastinal mass with higher accuracy.

Keywords: Anterior mediastinal masses, Middle mediastinal masses, Posterior mediastinal masses, Retrospective cross-sectional study.

(C) 2020 The Authors. Published by Innovare Academic Sciences Pvt Ltd. This is an open access article under the CC BY license (http://creativecommons. org/licenses/by/4. 0/) DOI: http://dx.doi.org/10.22159/ajpcr.2020.v13i8.38210

\section{INTRODUCTION}

The mediastinum is the cavity that differentiates the lungs from the remainder of the chest. It contains the esophagus, heart, thymus, trachea, and aorta. The mediastinum has three chief parts named as the anterior mediastinum (front), the middle mediastinum, and the posterior mediastinum (back). Anatomically mediastinum is limited by the thoracic inlet, pleural cavity, and diaphragm [1]. Mediastinal masses are generally uncommon (approx. 3\%) of tumors found in the chest region. The most common mass present in the anterior mediastinum is thymoma, teratoma, and lymphoma. The most common congenital cysts and neurogenic masses are formed in the middle and posterior mediastinum [2]. Almost $40 \%$ of the people are having mediastinal tumors with no symptoms. Most of the masses are frequently found on a chest X-ray that is performed for another reason. When symptoms are present, they are often as a result of the compression of surrounding structures, such as the spinal cord, heart, or pericardium [3].

Before the computed tomography (CT) machine was discovered, only chest radiographs are the modality that can evaluate the pathology present in mediastinum. However, now computed tomography has become the principal modality for the assessment of mediastinal masses, the involvement of surrounding structure and characteristics of various lesions present in the mediastinum. CT has the ability to differentiate density and also provides a three-dimensional crosssectional view of anatomic relations. CT has better spatial resolution and shorter imaging time as compared to magnetic resonance (MR) imaging, besides it is less expensive and more widely available than MR [4-6]. CT is the best modality of choice by which we can precisely assess of nature of the mass and easily detect fat and calcium within the tumor mass. CT also the pre-operative imaging technique, which enables the volumetric images of the chest and facilitates accurate evaluation using post-processing techniques such as minimum intensity projection and volume rendering [7,8]. CT is a more appropriate method of imaging to base the various diagnoses of mediastinal pathologies on direct monitoring of structure and tissue from which the pathology is arising. CT gives more clear delineation of pathologies and differentiates those better form normal tissues. The main focus of this study is to evaluate the sensitivity and specificity of multidetector CT (MDCT) for the localization and estimation of the age group where the occurrence of a mediastinal mass is most common.

\section{METHODS}

A retrospective cross-sectional study included 33 male and 21 female samples in the age range between 20 to 70 years were taken. The raw data were taken from the CT console, specifically from the patient suspected of mediastinal masses. The inclusion criteria were the patient scheduled for CT thorax with clinically suspected cases of mediastinal mass during the study period. The exclusion criteria were traumatic injury and any cardiac cases. Chest CT was performed on the 128 slice Philips Genuity machine with a normal daily protocol. The research was conducted between August 2017 to March 2018. The patient is positioned on the CT couch with the supine position with the area coverage from the lung apices to the domes of the diaphragm. The topogram of the thorax was acquired in the anteroposterior position. There is no gantry angulation during the examination of the thorax. The protocol which was taken for CT thorax is as follows: Collimation of $128 \times 0.625$, FOV $350 \mathrm{~mm}$, tube voltage of $120 \mathrm{kVp}$, tube current $101 \mathrm{mAs}$, and scan times $6.4 \mathrm{~s}$. A scan was acquired with a slice thickness of $5 \mathrm{~mm}$ and an increment of $5 \mathrm{~mm}$ with the filter Standard B. For elimination of motion artifacts, patients were strictly instructed before the scan. To perform the study, inform consent from each patient was taken. 


\section{Data analysis}

The data were collected to see the localization, age distribution, and gender distribution of mediastinal masses. The data were analyzed using Microsoft Excel 2010 using descriptive statistical tools such as frequencies, mean, standard deviation, and percentages.

\section{RESULTS}

A total of 54 samples were included in the study. Out of 54 samples, 33 males and 21 females in the age range between 20 to 70 years, and the overall mean was found to be $47.03 \pm 10.41$. During this study period, patients who were referred for CT scan of the chest in the department of radiodiagnosis with a clinical suspicion of a mediastinal mass or who had an abnormal chest radiograph suggestive of mediastinal mass were included in the study. The study revealed that out of 54 patients, 23 patients, i.e., $42.5 \%$ patients had anterior mediastinal mass, 11 patients, i.e., $20.5 \%$ of patients had middle mediastinal mass and rest 20 patients, i.e., $37 \%$ of patient had a mediastinal mass in the posterior side of the chest (Table 1).

The highest number of patients, i.e., 21 patients fall within the age group of 40-49 years where 13 patients were male and 8 patients were female which shows that in this age group the frequency of occurring the mediastinal masses is much higher, followed by the age group of $50-59$ years, i.e., 18 patients where 13 patients were male and 5 patients were female. In the age group of 40-49 years and 50-59 years clearly show that the male is having the highest probability of occurrence of mediastinal masses compare to other age groups of males. Likewise, the age group of 40-49 years shows that the females in that age group have the highest probability of occurrence of mediastinal masses compare to other age groups of females (Table 2).

\section{DISCUSSION}

The mediastinum is the common site of high ranges of diseases such as cysts, benign tumors, malignant tumors, lymph nodes, and vascular lesions. CT is the modality of choice to identify the precise location, characterization, and extent of these masses. Kaur et al. conducted a study based on mediastinal masses in which $53 \%$ of the patients having the symptoms of cough, followed by $50 \%$ dyspnea, $20 \%$ chest pain, and $17 \%$ fever [9]. Another study performed by Davis et al., in which 30\% of the patients having the normal symptoms of chest pain and $20 \%$ are suffering from fever [10]. Similarly, in the present study, the majority, i.e., $46 \%$ of the patient is having symptoms of cough and almost $23 \%$ chest pain. Mediastinal masses are uncommon cases, where $50 \%$ of cases were asymptomatic.

Strollo et al., 1997, performed a study based on tumors evaluation in the anterior mediastinum; in this project, anterior mediastinum comprised $50 \%$ of all the mediastinal masses [11]. A similar study

Table 1: Compartmental distribution of mediastinal masses

\begin{tabular}{lll}
\hline Mediastinal compartment & Number of cases & Percentage \\
\hline Anterior & 23 & 42.5 \\
Middle & 11 & 20.5 \\
Posterior & 20 & 37 \\
Total & 54 & 100 \\
\hline
\end{tabular}

Table 2: Distribution of patients examined according to the gender and age group

\begin{tabular}{llll}
\hline Age group & $\begin{array}{l}\text { Total number of cases } \\
\text { (no. 54) }\end{array}$ & $\begin{array}{l}\text { Male } \\
\text { (no. 33) }\end{array}$ & $\begin{array}{l}\text { Female } \\
\text { (no. 21) }\end{array}$ \\
\hline $20-29$ & 3 & 1 & 2 \\
$30-39$ & 8 & 4 & 4 \\
$40-49$ & 21 & 13 & 8 \\
$50-59$ & 18 & 13 & 5 \\
$60-69$ & 3 & 1 & 2 \\
$70-79$ & 1 & 1 & 0 \\
\hline
\end{tabular}

performed by Takeda et al. in Japan which is based on the evaluation of mediastinal mass in adult and pediatric population in which the most prevalent location was the anterior compartment, i.e., $68 \%$ in case of adults and $52 \%$ in the posterior compartment in case of children [12]. Another project reported by Kumar et al., which is based on mediastinal masses in which the age group of samples ranges from 7 to 80 years was included and $86 \%$ cases having the involvement of anterior mediastinal masses [13], whereas, in our present study, $42.5 \%$ of mediastinal masses were found in the anterior mediastinum, followed by $37 \%$ in the posterior mediastinum and $20.5 \%$ lesion found in the middle mediastinum which is similar to the study conducted by Davis et al., $54 \%$ mass are found in the anterior mediastinum, followed by $26 \%$ in the posterior mediastinum and $20 \%$ in the middle mediastinum [10]. Hence, it concluded that the majority of the masses were seen in the anterior compartment, followed by the posterior and middle.

Sones et al. concluded a study based on CT evaluation of intra-thoracic masses in which they revealed that CT is the modality of choice to demonstrate mediastinal pathology in almost all the cases without any fail [14]. A similar study reported by Glazer et al., which depicted that CT can be useful to determine the mediastinal lesion precisely [15]. Kaur et al. performed a study similar to our study in which they revealed that maximum numbers of cases, i.e., $20 \%$ were seen in the age group of 51-60 years [9]. In the present study, the result shows that the CT can detect mediastinal pathology precisely and the participants having the age range of 40-49 years category have a higher frequency of occurring mediastinal pathology, followed by the age range 50-59 years and 30-39 years.

In our study, most often masses found in the anterior mediastinum are lymphomas and thymomas. In the middle mediastinum and posterior mediastinum aneurysms, lymph node masses and nerve sheath tumors are most common. Trans-compartmental involvement is seen in lymph node masses and aneurysms. The present study revealed that the CT scan can be helpful in differentiating the precise location of mediastinal masses.

\section{CONCLUSION}

Chest X-ray is the initial modality in suspected cases of mediastinal pathology, but further assessment required MDCT for accurate localization of pathology, scrutinize their morphology, involvement of adjacent structures, and associated findings in lungs, pleura, and chest wall. In our study, the findings suggested that the forepart of the mediastinum, i.e., anterior compartment is the most common compartment involved, followed by the posterior and middle compartment. Most of the masses present in the mediastinum are clearly defined, showing heterogeneous enhancement after injection of contrast medium in contrast scan. Finally, we concluded that, in our present study, MDCT is an important modality that can evaluate the mediastinal masses with higher accuracy.

\section{AUTHORS' CONTRIBUTIONS}

The study was designed by Himal Rai, Manna Debnath, Anand P. Verma, and the raw data were collected by Himal Rai. The manuscript is drafted by Manna Debnath and Himal Rai. All the authors have read and approved the manuscript.

\section{CONFLICTS OF INTEREST}

The authors declare that there are no conflicts of interest regarding the publication of this article.

\section{AUTHORS' FUNDING}

None.

\section{REFERENCES}

1. Whitten CR, Khan S, Munneke GJ, Grubnic S. A diagnostic approach to mediastinal abnormalities. Radiographics 2007;27:657-71.

2. Duwe BV, Sterman DH, Musani AI. Tumors of the mediastinum. Chest 
2005;128:2893-909.

3. Trupiano JK, Rice TW, Herzog K, Barr FG, Shipley J, Fisher C, et al. Mediastinal synovial sarcoma: Report of two cases with molecular genetic analysis. Ann Thorac Surg 2002;73:628-30.

4. Dutta P, Patel KK, Choudhury PR. Mediastinal masses: A radiologica 1 study of 50 cases. IJBR 2014;5:468-73.

5. Zope AM, Kachewar SG, Ghule SS, Lakhkar DL. Computed tomographic evaluation of mediastinal masses. Sch J App Med Sci 2016;4:4388-93.

6. Shashikumar MR., Kumar NL, Rajendra CP, Nanjaraj CN, Kumar P, Manupratap P, et al. Computed tomographic evaluation of mediastinal masses. JEMDS 2015;4:9951-62.

7. Visakh T. Variation in the size of abdominal aorta measured on computed tomography. Asian J Pharm Clin Res 2018;11:146-8.

8. Fernandes SF, Pradhan A. Estimation of subcarinal angle using minimum intensity projection in computed tomography. Asian J Pharm Clin Res 2018;11:383-5.

9. Kaur H, Tiwari P, Dugg P, Ghuman J, Shivhare P, Mehmi RS. Computed tomographic evaluation of mediastinal masses/lesions with contrast enhancement and correlation with pathological diagnosis-a study of 120 cases. J Biomed Graph Comput 2014;4:28

10. Davis RD, Oldham HN, Sabiston DC. Primary cysts and neoplasms of the mediastinum: Recent changes in clinical presentation, methods of diagnosis, management, and results. Ann Thorac Surg 1987;44:229-37.

11. Strollo DC, de Christenson ML, Jett JR. Primary mediastinal tumors. Part 1: Tumors of the anterior mediastinum. Chest 1997;112:511-22.

12. Takeda SI, Miyoshi S, Akashi A, Ohta M, Minami M, Okumura M, et al. Clinical spectrum of primary mediastinal tumors: A comparison of adult and pediatric populations at a single Japanese institution. J Surg Oncol 2003;83:24-30.

13. Kumar S, Pande S, Baghel P, Bhargav OP. Radiological profile of mediastinal masses with special reference to computed tomography. J Evol Med Dent Sci 2015;4:16280-6.

14. Sones PJ Jr., Torres WE, Colvin RS, Meier WL, Sprawls P, Rogers JV Jr. Effectiveness of CT in evaluating intrathoracic masses. Am J Roentgenol 1982;139:469-75.

15. Glazer HS, Siegel MJ, Sagel SS. Low-attenuation mediastinal masses on CT. Am J Roentgenol 1989;152:1173-7. 